\section{Acknowledgments.}

Financial support. Dr Munster is supported by the Intramural Research Program of the National Institute of Allergy and Infectious Diseases, National Institutes of Health.

Conflicts of interest. The authors declare no conflicts of interest.

\section{References}

1. Judson SD, Munster VJ. Nosocomial transmission of emerging viruses via aerosol-generating medical procedures. Viruses. 2019;11:940.

2. Chowell G, Abdirizak F, Lee S, et al. Transmission characteristics of MERS and SARS in the healthcare setting: a comparative study. BMC Med 2015; 13:210.

3. Xu D, Zhang Z, Jin L, et al. Persistent shedding of viable SARS-CoV in urine and stool of SARS patients during the convalescent phase. Eur J Clin Microbiol Infect Dis 2005;24:165-171.
4. van Doremalen N, Bushmaker T, Munster V. Stability of Middle East respiratory syndrome coronavirus (MERS-CoV) under different environmental conditions. Eurosurveillance 2013;18:20590.

5. Corman VM, Albarrak AM, Omrani AS, et al. Viral shedding and antibody response in 37 patients with Middle East respiratory syndrome coronavirus infection. Clin Infect Dis 2015;62(4):civ951.

6. Wu Z, McGoogan JM. Characteristics of and important lessons from the coronavirus disease 2019 (COVID-19) outbreak in China. JAMA 2020;323: 1239-1242.

7. Livingston E, Bucher K. Coronavirus Disease 2019 (COVID-19) in Italy. JAMA 2020;323:1335.

8. Wang W, Xu Y, Gao R, et al. Detection of SARS-CoV-2 in different types of clinical specimens. JAMAh 2020;323:1843-1844.

9. van Doremalen N, Bushmaker T, Morris DH, et al. Aerosol and surface stability of SARS-CoV-2 as compared with SARS-CoV-1. N Engl J Med 2020;382:1564-1567.

10. Guo Z-D, Wang Z-Y, Zhang S-F, et al. Aerosol and surface distribution of severe acute respiratory syndrome coronavirus 2 in hospital wards, Wuhan, China, 2020. Emerg Infect Dis. 2020;26(7). doi: 10.3201/eid2607.200839.

\title{
Adverse effects of nasopharyngeal swabs: Three-dimensional printed versus commercial swabs
}

\author{
Kalpana Gupta MD, MPH ${ }^{1,2}$ (1), Pamela M. Bellino OTR/L, CPPS ${ }^{1}$ and Michael E. Charness MD ${ }^{1,2,3}$ \\ ${ }^{1}$ VA Boston Healthcare System, West Roxbury, Massachusetts, ${ }^{2}$ Boston University School of Medicine, Boston, Massachusetts and ${ }^{3} \mathrm{Harvard}$ Medical School, \\ Boston, Massachusetts
}

To the Editor-To date, $>6$ million tests for COVID-19 have been performed in the United States, with the vast majority utilizing nasopharyngeal sampling. ${ }^{1}$ The need for large-scale testing in the COVID-19 pandemic has created a global shortage of commercial nasopharyngeal swabs. One approach to this shortage has been the 3-dimensional (3D) printing of nasopharyngeal swabs. Swabs printed on a 3D printer (3D swab) differ somewhat from commercially produced swabs: they having larger heads, less flexibility, and a plastic rather than cotton or polyester fiber tip. These 3D swabs are class 1 medical devices, and their diagnostic efficacy has been validated through field testing. ${ }^{2}$

Guidance on the safe collection of nasopharyngeal samples using commercial swabs is available in text and video format ${ }^{3,4}$; however, no data are available on the adverse effects of either commercial or 3D swabs, making it difficult to assess their relative safety. To expand testing at our medical center, we printed the Northwell prototype 3D swab using specifications obtained from the technology transfer office at the University of South Florida. As part of our safety assessment of this prototype, we identified adverse effects of NP swabbing in employees using both commercial and 3D swabs. Epistaxis occurred immediately or shortly following the removal of the swab in $5.0 \%$ of employees tested with the $3 \mathrm{D}$ swab and in $8.3 \%$ of employees tested with the commercial swab (Table 1). Epistaxis was usually mild and self-limited, although 1 employee required an emergency department visit for ongoing

Author for correspondence: Kalpana Gupta, E-mail: Kalpana.gupta@va.gov Cite this article: Gupta K, Bellino PM, and Charness ME. (2021). Adverse effects of nasopharyngeal swabs: Three-dimensional printed versus commercial swabs. Infection Control \& Hospital Epidemiology, 42: 641-642, https://doi.org/10.1017/ice.2020.297
Table 1. Comparison of 3D Printed Nasopharyngeal Swabs Versus Commercial Swabs

\begin{tabular}{lcc}
\hline Variable & Commercial Swab, No. & 3D Swab, No. \\
\hline Sample size & 96 & 80 \\
\hline Epistaxis, no. (\%) & $8(8.3)$ & $4(5.0)$ \\
\hline Nasal discomfort & 4 & 6 \\
\hline Headache & 5 & 2 \\
\hline Ear discomfort & 5 & 1 \\
\hline Rhinorrhea & 5 & 1 \\
\hline
\end{tabular}

epistaxis after testing with a commercial swab. Other minor adverse effects included nasal discomfort, headache, earache, and rhinorrhea, which typically lasted hours to a day.

Our finding that epistaxis is equally common after the use of $3 \mathrm{D}$ and commercial swabs provides reassurance that $3 \mathrm{D}$ swabs are a safe alternative to commercial swabs. However, the $5 \%-10 \%$ incidence of epistaxis after nasal swabbing with either commercial or 3D swabs warrants caution in testing individuals at increased risk for bleeding. Nursing home residents have been disproportionately affected by COVID-19, and a recent point prevalence study of Medicare fee-for-service beneficiaries found that almost half of 37,787 nursing home residents were treated with oral anticoagulants. ${ }^{5}$ Rates of epistaxis after nasal swabbing should be studied in larger populations, including the elderly, and individuals at increased bleeding risk should be monitored after the procedure. Fortunately, less invasive methods of SARS-CoV-2 
detection, such as midturbinate or saliva sampling, are on the horizon.

Acknowledgments. We are grateful to Michael Kulig, Elena Buckley, Andrew Risio, Jennifer Bryant, and Dr. Steven Brecher for their efforts in implementing our 3D-printing of nasopharyngeal swabs.

Financial support. No financial support was provided relevant to this article.

Conflicts of interest. All authors report no conflicts of interest relevant to this article.

\section{References}

1. The COVID tracking project website. https://covidtracking.com/. Accessed May 9, 2020.
2. Callahan CJ, Lee R, Zulauf KE, et al. Open development and clinical validation of multiple 3D-printed nasopharyngeal collection swabs: rapid resolution of a critical COVID-19 testing bottleneck. J Clin Micro May 2020 [Epub ahead of print]. doi: 10.1128/JCM.00876-20.

3. Nasal (anterior nasal) specimen collection for SARS-CoV-2 diagnostic testing. Centers for Disease Control and Prevention website. https:/www.cdc.gov/ coronavirus/2019-ncov/downloads/OASH-nasal-specimen-collection-factsheet.pdf. Accessed May 9, 2020.

4. Marty F, Chen K, Verrill KA. How to obtain a nasopharyngeal swab specimen. N Engl J Med 2020;382(22):e76. doi: 10.1056/NEJMvcm2010260.

5. Alcusky M, McManus DD, Hume AL, Fisher M, Tjia J, Lapane KL. Changes in anticoagulant utilization among United States nursing home residents with atrial fibrillation from 2011 to 2016. J Am Heart Assoc. 2019;8(9): e012023. doi: 10.1161/JAHA.119.012023.

\title{
Pediatric antimicrobial stewardship in the COVID-19 outbreak
}

\author{
Eneritz Velasco-Arnaiz MD ${ }^{1,2}$ (1), Maria Goretti López-Ramos PharmD, BCPPS ${ }^{1,3}$, Silvia Simó-Nebot MD ${ }^{1,2,4}$, \\ lolanda Jordan MD, $\mathrm{PhD}^{1,4,5,6,7}$, María Ríos-Barnés MD ${ }^{1,2}$, Mireia Urrea-Ayala MD, $\mathrm{PhD}^{1,8}$, Manuel Monsonís BSc ${ }^{1,9}$, \\ Clàudia Fortuny MD, $\mathrm{PhD}^{2,4,6,7}$ and Antoni Noguera-Julian MD, $\mathrm{PhD}^{2,4,6,7}$ on behalf of the Kids Corona project \\ ${ }^{1}$ Sant Joan de Déu Antimicrobial Stewardship Program (SJD-ASP), Sant Joan de Déu Hospital, Barcelona, Spain, ${ }^{2}$ Infectious Diseases Unit, Department of \\ Pediatrics, Sant Joan de Déu Hospital, Barcelona, Spain, ${ }^{3}$ Pharmacy Department, Sant Joan de Déu Hospital, Barcelona, Spain, ${ }^{4}$ Centre for Biomedical Network \\ Research on Epidemiology and Public Health (CIBERESP), Madrid, Spain, ${ }^{5}$ Pediatric Intensive Care Unit, Sant Joan de Déu Hospital, Barcelona, Spain, \\ ${ }^{6}$ Department of Pediatrics, University of Barcelona, Barcelona, Spain, ${ }^{7}$ Red de Investigación Translacional en Infectología Pediátrica, RITIP, Madrid, Spain, \\ ${ }^{8}$ Infection Control Department, Sant Joan de Déu Hospital, Barcelona, Spain and ${ }^{9}$ Clinical Microbiology Department, Sant Joan de Déu Hospital, \\ Barcelona, Spain
}

To the Editor-Growing evidence supports the positive impact of antimicrobial stewardship programs (ASPs) on antimicrobial use, including pediatrics. ${ }^{1}$ Although short of the level of acceptance these have reached in the United States, the implementation of pediatric ASPs in European hospitals has increased over the last few years. ${ }^{1}$

It has been suggested that the ASP should be helpful in the preparation for and response to the SARS-CoV-2/COVID-19 outbreak, ${ }^{2}$ but no formal recommendations have been published. Whether pediatric ASP remains an essential activity or not during the COVID-19 pandemic has yet to be clarified. Here, we describe how the COVID-19 pandemic has impacted antimicrobial use in a referral pediatric hospital, and we propose a supporting role for ASP teams in the local management of the outbreak.

The first COVID-19 case in Catalonia, Spain, was reported on February 25, 2020. By mid-March, most pediatric and obstetrics departments in the region were shut to increase the capacity for adult COVID-19 patients. Hospital Sant Joan de Déu Barcelona (SJD) remained the largest pediatric and maternal referral center in the region. COVID-19 and non-COVID-19 pediatric and young adult patients were transferred to our wards and pediatric ICU (PICU), and the number of daily deliveries tripled, whereas all nonemergency clinical, teaching, and research activities were

Author for correspondence: Eneritz Velasco Arnaiz, E-mail: evelasco@ sjdhospitalbarcelona.org

Cite this article: Velasco-Arnaiz E, et al. (2021). Pediatric antimicrobial stewardship in the COVID-19 outbreak. Infection Control \& Hospital Epidemiology, 42: 642-644, https:// doi.org/10.1017/ice.2020.312 postponed. Compared to the same months in 2019, in March 2020 , total hospital stays decreased by $0.8 \%$ in the PICU and $15.2 \%$ in non-PICU areas, and in April 2020, total hospital stays decreased by $23.7 \%$ in the PICU and $22.2 \%$ in non-PICU areas.

Following institutional recommendations, the SJD-ASP ${ }^{3}$ team reduced on-site work, but they continued to provide specific recommendations on individual antimicrobial prescriptions upon consultation by prescribers, and they monitored systemic antibiotic and antifungal use: days of-therapy (DOT) per 100 days present (DP). From March 16 to April 30 2020, 210 randomly selected prescriptions were assessed for quality. ${ }^{3}$

Because SARS-CoV-2 is a viral infection, it is not expected to directly influence antibiotic or antifungal use beyond the use of antibiotics with possible antiviral effect (ie, azithromycin) ${ }^{4}$ and the use of broad-spectrum antibiotics for superinfection in severe COVID-19 patients. ${ }^{5}$ However, we also observed antimicrobial use changes indirectly related to the outbreak. Antimicrobial use in March and April 2020 was significantly higher than in the same months in 2019 (Table 1). As expected, the use of azithromycin, included as first-line therapy in severe COVID-19 patients in combination with hydroxychloroquine, increased, particularly in the PICU. The use of ceftriaxone and teicoplanin, which were also prescribed at admission in severe COVID-19 cases, doubled in the PICU in April 2020 compared with April 2019. Other than ceftriaxone, antibiotics for community-acquired infections were prescribed less than in the same period in 2019, and cefazolin use decreased due to the dramatic drop in the number of surgeries. In contrast, the use of most broad-spectrum anti-gram-negative

(c) 2020 by The Society for Healthcare Epidemiology of America. All rights reserved. This is an Open Access article, distributed under the terms of the Creative Commons Attribution licence (http://creativecommons.org/licenses/by/4.0/), which permits unrestricted re-use, distribution, and reproduction in any medium, provided the original work is properly cited. 